\title{
Parents as primary caregivers in the neonatal intensive care unit
}

\author{
Shoo K. Lee MBBS PhD, Karel O'Brien MB BCh BAO MSc
}

$\mathrm{T}$ he neonatal intensive care unit is highly stressful for parents, who are physically separated from their infants. They often feel unable to participate meaningfully in their infant's care and feel unprepared for discharge. ${ }^{1}$ To address this problem, family-centred neonatal intensive care programs have been developed to promote skin-to-skin care, involve parents in decision-making and caregiving, and provide education and psychological support. ${ }^{2}$ The aim of care-by-parent models is to improve the quality, continuity and consistency of neonatal care and ensure a smooth transition from hospital to home. Even though a high level of family involvement is generally accepted as optimal, ${ }^{3}$ relatively few neonatal care programs in units that provide level 3 care facilitate this approach. ${ }^{47}$ Box 1 describes the levels of care in Canadian neonatal intensive care units.

\section{How can parents be the primary caregivers for their infant in the neonatal intensive care unit?}

We are currently studying the Family Integrated Care program in 20 level 3 neonatal units across Canada, following a successful pilot study. ${ }^{6}$ Our program adapted the concepts of a rooming-in model developed in Tallinn, Estonia, for a level 3 neonatal care environment in Canada. We developed an education program for parents, designed a training module for nurses, ensured that parents had access to peer support, and improved the environment so that parents were able to spend 8-12 hours each day in the unit. Parents participate in information sharing during rounds. As parents' confidence grows, they become the primary caregivers for their infants, either continuously or for a substantial portion of their infant's stay in the intensive care unit.

Integration of parents into their infant's care begins at admission. Following orientation, parents are empowered to provide as much of their infant's care as they are able. This includes feeding, bathing, changing diapers, dressing, holding skin-to-skin, and administering oral medications. Parents take part in education sessions and are supported by the nursing staff to actively participate in daily decision-making, provide reports during rounds and record their infant's condition and progress (Figures 1 and 2). In addition to educating and guiding parents, nurses provide more specialized forms of care, such as administration of intravenous fluid and medications.

The provision of psychosocial and physical support is also important and can include support from other families in the neonatal unit or from a volun-

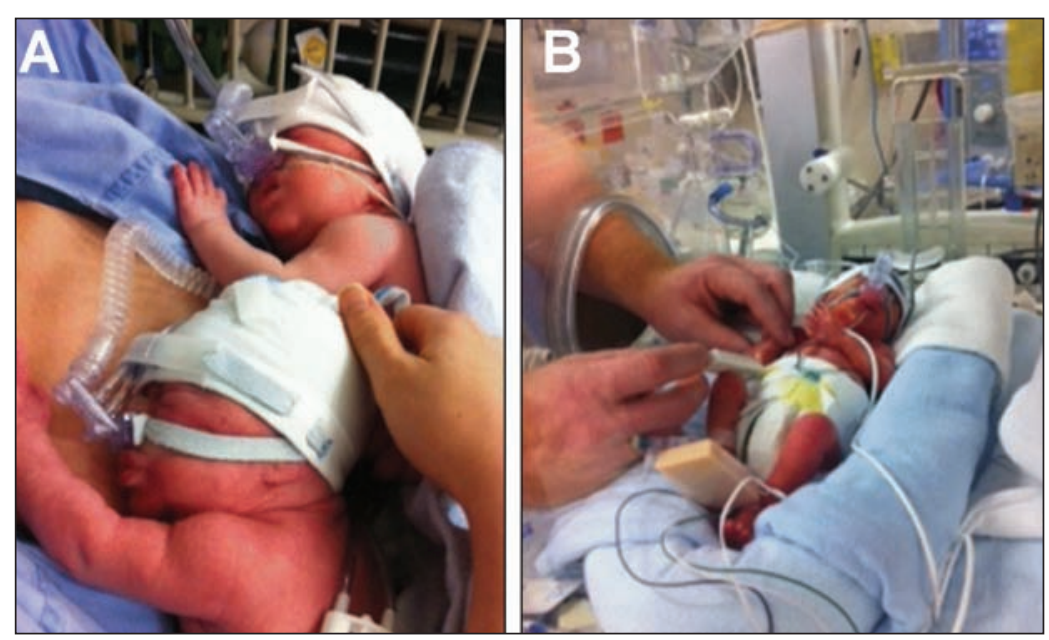

Figure 1: (A) A mother in the Family Integrated Care (care-by-parent) program providing skin-to-skin care for her twins. (B) A father measuring his infant's temperature.

\section{KEY POINTS}

- Having parents provide most of their infant's care in the neonatal intensive care unit has the potential to improve short- and long-term outcomes among infants and reduce stress among parents.

- Care-by-parent programs in neonatal units that provide level 2 care are becoming more common, but there is very little opportunity for parents to participate in their infant's care in level 3 neonatal units.

- The effect of care-by-parent programs in level 3 neonatal units is an ongoing area of research. 
teer parent "buddy" with relevant experience. Physical support includes ensuring that the intensive care environment is conducive to parents spending prolonged periods of time there. This could include providing a comfortable chair by each infant's bedside, a lounge in which parents can prepare food or relax, and bedrooms for the parents if rooming-in is not possible. Responsibilities of the nursing staff and parents are formalized to ensure that the nursing staff remain responsible for both infant care and oversight of the care provided by the parents.

\section{What are the benefits of this model of care?}

Care-by-parent programs in level 2 and 3 neonatal units have been reported to reduce the length of stay by 3.2-5.3 days, ${ }^{7,9,10}$ decrease hospital readmission, ${ }^{5}$ and improve infant outcomes, such as the rate of breastfeeding and weight gain, ${ }^{6}$ in cohort studies and one randomized controlled trial. Although the long-term effects of care-byparent programs have not been investigated,

\section{Box 1: Levels of neonatal care in Canada}

The tiered provision of neonatal care in Canada closely follows the American Academy of Pediatrics ${ }^{8}$ definitions of care.

Level 1: Basic care is provided to low-risk healthy infants in a mother-andbaby unit.

Level 2: Care is provided to stable or moderately ill infants born after 32 weeks' gestation with problems that are expected to resolve rapidly. The unit in which level 2 care is provided is referred to as a level 2 neonatal unit, level 2 neonatal intensive care unit, or a special-care nursery.

Level 3: Care is provided to critically ill infants born before 32 weeks' gestation or with significant medical or surgical conditions, including the need for mechanical ventilation and other life support. The unit that provides level 3 care is referred to as a level 3 neonatal unit or a level 3 neonatal intensive care unit.

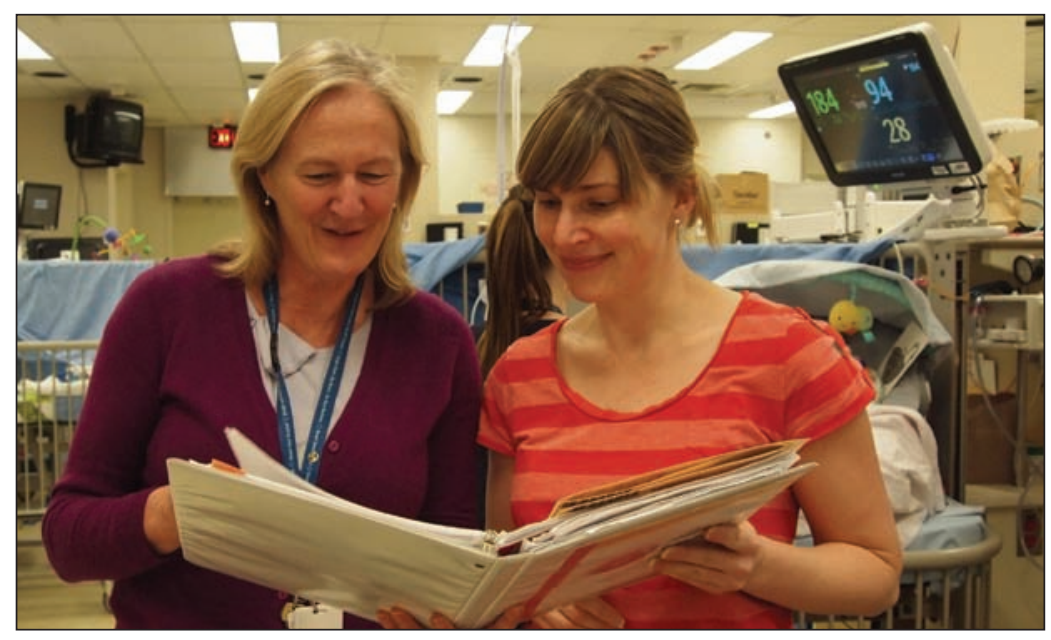

Figure 2: In the care-by-parent model, parents are provided with education, including how to keep track of their infant's progress, so that they can actively participate in daily decision-making. increased parent education and interaction between parents and their preterm infant was reported to improve infant behaviour and longterm cognitive development in a cohort study ${ }^{11}$ and randomized controlled trial. ${ }^{12}$ Parents are also likely to benefit from this care model, because their stress levels may be reduced and they are better equipped to care for their infant after discharge. ${ }^{6}$ For the Family Integrated Care program, our pilot study of 31 infants and their mothers showed a significantly improved rate of weight gain among infants, an $80 \%$ increase in breastfeeding at discharge, and a $25 \%$ reduction in parental stress compared with retrospective controls (i.e., infants who were not enrolled in the program). All parents and nurses surveyed reported positive experiences. ${ }^{7}$

\section{What are the possible harms associated with this model of care?}

It is possible that the parents may not detect changes in their infant's condition that require prompt medical attention. However, nurses retain primary responsibility for infants and supervise parents closely, which should ensure that appropriate care is given. Another concern is that parents may become overly anxious about providing care for their sick infant. However, in our experience, most parents report decreased anxiety and stress because they feel in control and wellinformed, and they have a purposeful role in caring for their infant. We observed no adverse events as a result of the provision of care by parents in our pilot study. ${ }^{6}$

\section{What are the barriers to implementing this type of program?}

Encouraging mothers to room-in with their infant is becoming more common in neonatal units that provide level 2 care, particularly in settings with limited resources. ${ }^{4}$ However, infants in these units are those at moderate risk of serious complications who have problems that are expected to resolve rapidly. ${ }^{8}$ For critically ill infants in level 3 units, who may require advanced respiratory care or other complex treatments ${ }^{8}$ and where parents are not able to roomin, parental involvement in caregiving is more challenging.

Having parents as the primary caregivers in a level 3 neonatal unit represents a substantial shift in the current North American model of neonatal care. As such, there are numerous barriers to 
widespread implementation of this model of care. There needs to be further study of the effects of such programs on short- and long-term infant outcomes, parent mental health, family economics and stability, and costs to the health care system. Further exploration is needed into the level of involvement that parents can have in the care of extremely sick infants who require mechanical ventilation.

\section{What can we expect in the future?}

Because our pilot study showed that the care-byparent model is feasible in a level 3 neonatal unit and has the potential to improve infant and parent outcomes, we are now conducting a cluster randomized controlled trial in 20 level 3 neonatal units across Canada. If our study confirms that this model has positive outcomes, we will work to have the practice adopted as standard of care for all level 3 neonatal units in Canada. The responses by parents and staff in the units that have implemented our program have been very positive, and we expect uptake to be high. Efforts are ongoing in both developed and developing countries, and we expect that the move to empower parents to fully participate in their infant's care in both level 2 and level 3 neonatal units will continue to expand.

\section{References}

1. Scherf RF, Reid KW. Going home: what NICU nurses need to know about home care. Neonatal Netw 2006;25:421-5.

2. Gooding JS, Cooper LG, Blaine AI, et al. Family support and family-centered care in the neonatal intensive care unit: origins, advances, impact. Semin Perinatol 2011;35:20-8.

3. American Academy of Pediatrics Committee on Hospital Care, Institute For Patient- and Family-Centered Care. Patient- and family-centered care and the pediatrician's role [policy]. Pediatrics 2012;129:394-404.

4. Bhutta ZA, Khan I, Salat S, et al. Reducing length of stay in hospital for very low birthweight infants by involving mothers in a stepdown unit: an experience from Karachi (Pakistan). BMJ 2004; 329:1151-5.

5. Erdeve $\mathrm{O}$, Arsan $\mathrm{S}$, Yigit $\mathrm{S}$, et al. The impact of individual room on rehospitalization and health service utilization in preterms after discharge. Acta Paediatr 2008;97:1351-7.

6. O'Brien K, Bracht M, Macdonell K, et al. A pilot cohort analytic study of Family Integrated Care in a Canadian neonatal intensive care unit. BMC Pregnancy Childbirth 2013;13(Suppl 1):S12.

7. Ortenstrand A, Westrup B, Brostrom EB, et al. The Stockholm Neonatal Family Centered Care study: effects on length of stay and infant morbidity. Pediatrics 2010;125:e278-85.

8. American Academy of Pediatrics Committee on Fetus and Newborn. Levels of neonatal care. Pediatrics 2012;130:587-97.

9. Chabaud F, David-Tchouda S, Belin V, et al. Influence of hospital location on short-term fate of premature infants born at 34 weeks of gestation [article in French]. Arch Pediatr 2012;19: 391-5.

10. Narayanan I, Kumar H, Singhal PK, et al. Maternal participation in the care of the high risk infant: follow-up evaluation. Indian Pediatr 1991;28:161-7.

11. Achenbach TM, Howell CT, Aoki MF, et al. Nine-year outcome of the Vermont intervention program for low birth weight infants. Pediatrics 1993;91:45-55.

12. Melnyk BM, Alpert-Gillis L, Feinstein NF, et al. Improving cognitive development of low-birth-weight premature infants with the COPE program: a pilot study of the benefit of early NICU intervention with mothers. Res Nurs Health 2001;24:373-89.

Affiliation: Department of Paediatrics and Maternal Infant Care Research Centre, Mount Sinai Hospital, University of Toronto, Toronto, Ont.

Contributors: Both of the authors wrote and revised the article and approved the final version submitted for publication.

Acknowledgements: The authors thank Ruth Warre from the Maternal-Infant Care Research Centre for providing editorial support in the preparation of this article.

The Maternal-Infant Care Research Centre is supported by the Ontario Ministry of Health and Long-Term Care.

Funding for the pilot study was provided by the Preterm Birth and Health Outcomes Team, Alberta Innovates, and the Ontario Ministry of Health and Long-Term Care. The Canadian Institutes of Health Research and the Ontario Ministry of Health and Long-Term Care have provided funding for the randomized controlled trial of the Family Integrated Care program. 